\title{
ON ESSENTIALLY ISOMETRIC CONFORMAL TRANSFORMATION GROUPS
}

\author{
By Hitosi Hiramatu
}

A conformal transformation group $G$ of a Riemannian manifold $(\mathfrak{B}, g), \mathfrak{B}$ being a manifold and $g$ a Riemannian metric tensor field on $\mathfrak{V}$, is said to be essentially isometric, if there exists a positive-valued function $f$ on $\mathfrak{B}$ such that $G$ is an isometry group of another Riemannian manifold $(\mathfrak{B}, f g)$. Throughout this paper, we assume that manifolds, functions, vector fields and tensor fields are differentiable and of class $C^{\infty}$, Riemannian manifolds are connected and conformal transformation groups are connected and effective. The purpose of the present paper is to prove some theorems concerning essentially isometric conformal transformation groups.

For a Riemannian manifold $(\mathfrak{B}, g)$ of dimension $n$, we denote by $K, S$ and $k$ respectively the curvature tensor, the Ricci tensor and the curvature scalar. We consider two tensor fields $C$ and ' $C$ defined respectively by

$$
\begin{gathered}
C(X, Y) Z=K(X, Y) Z+\frac{1}{n-2}[L(X, Z) Y-L(Y, Z) X+g(X, Z) l(Y)-g(Y, Z) l(X)], \\
{ }^{\prime} C(X, Y, Z)=\left(\nabla_{X} S\right)(Y, Z)-\left(\nabla_{Y} S\right)(X, Z),
\end{gathered}
$$

$X, Y$ and $Z$ being arbitrary vector fields, where $L$ and $l$ are tensor fields defined respectively by

$$
\begin{gathered}
L(X, Y)=S(X, Y)-\frac{k}{2(n-1)} g(X, Y), \\
g(l(X), Y)=L(X, Y) .
\end{gathered}
$$

When $n>3$ (resp. when $n=3$ ), we call $C$ (resp. 'C) the Weyl conformal curvature tensor of $(\mathfrak{B}, g)$.

The following theorems concerning essentially isometric conformal transformation groups are well-known.

TheOREM A (Hlavatý [3]). If the Weyl conformal curvature tensor field of a Riemannian manifold $(\mathfrak{B}, g), \mathfrak{B}$ being of dimension $>2$, is not zero at every point of $\mathfrak{B}$, then any conformal transformation group of $(\mathfrak{V}, g)$ is essentially isometric.

THEOREM B (Ishihara [4]). If a conformal transformation group $G$ of $a$ Riemannian manifold is compact, then $G$ is essentially isometric.

Received April 28, 1971. 
We now consider a connected and effective Lie transformation group $G$ of a manifold $\mathfrak{B}$ and denote by $\mathbb{B}$ the Lie algebra of $G$. To an element $X$ of $\mathbb{S}$ there corresponds canonically a vector field $\tilde{X}$ on $\mathfrak{B}$ and the correspondence is an isomorphism of $\mathbb{B}$ onto a Lie algebra $\mathbb{B}$ consisting of $\tilde{X}$ corresponding to $X$ of $\mathbb{B}$. We denote by $\mathcal{L}_{\mathbb{X}^{*}}$ the Lie derivation with respect to $\tilde{X}$. The group $G$ is a conformal transformation group of a Riemannian manifold $(\mathfrak{B}, g)$ if and only if

$$
\mathcal{L}_{\tilde{X}} g=2 \phi_{\tilde{X}} g
$$

for any element $X$ of $\mathbb{B}, \phi_{\tilde{X}}$ being a function on $\mathfrak{B}$ which is called the function associated with $\tilde{X}$. The group $G$ is an isometry group of $(\mathfrak{B}, g)$ if and only if

$$
\mathcal{L}_{\tilde{X}} g=0
$$

for any element $X$ of $\$ 3$.

We now prove the following

LEMMA 1. A necessary and sufficient condition that a conformal transformation group $G$ of a Riemannian manifold $(\mathfrak{B}, g)$ be essentially isometric is that there exists a positive-valued function $f$ on $\mathfrak{B}$ satisfying

$$
\mathcal{L}_{\tilde{X}} f=-2 \phi_{\tilde{X}} f
$$

for any element $X$ of $\mathbb{S}$, where $\mathbb{S}$ is the Lie algebra of $G$ and $\phi_{\tilde{X}}$ is the function associated with $\tilde{X}$.

Proof. Suppose that $G$ is essentially isometric. Then there exists a positivevalued function $f$ on $\mathfrak{B}$ such that

$$
\mathcal{L}_{\tilde{\boldsymbol{X}}}(f g)=0
$$

for any element $X$ of $\mathbb{S}$, from which we have (2) by virtue of (1). Conversely, suppose that the relation (2) holds for some positive-valued function $f$ on $\mathfrak{B}$ and for any element $X$ of 8 . Then, from (1) and (2), we have (3) which means that $G$ is essentially isometric.

LeMma 2. Assume that a conformal transformation group $G$ of a Riemannian manifold $(\mathfrak{B}, g)$ is essentially isometric. If $G$ contains a Lie subgroup $M$ which is a transitive isometry group of $(\mathfrak{B}, g)$, then $G$ is necessarily an isometry group of $(\mathfrak{B}, g)$.

Proof. By Lemma 1, there exists a positive-valued function $f$ on $\mathfrak{B}$ satisfying (2). If we denote by $\mathfrak{M}$ the Lie algebra of the Lie subgroup $M$ which is transitive and isometric, then we have

$$
\mathcal{L}_{\tilde{\mathbf{X}}} \mid f=0
$$

for any element $X$ of $\mathfrak{M}$ because $\phi_{\tilde{X}}=0$. On the other hand, since $\mathfrak{B}$ is connected and $M$ is transitive, it follows from (4) that $f$ is a positive constant on $\mathfrak{F}$. Conse- 
quently we have the relation (4) for any element $X$ of $\$ \$ \&$, being the Lie algebra of $G$. Therefore, from (2), we have $\phi_{\tilde{X}}=0$ which means that $G$ is an isometry group.

From Theorem A and Lemma 2, we have

Theorem 1. Let $(\mathfrak{B}, g)$ be a Riemannian manifold of dimension $>2$, whose Weyl conformal curvature tensor field vanishes nowhere. If a conformal transformation group $G$ of $(\mathfrak{B}, g)$ contains a Lie subgroup which is a transitive isometry group of $(\mathfrak{B}, g)$, then $G$ is necessarily an isometry group.

We next prove the following

LEMMA 3. If a conformal transformation group $G$ of a Riemannian manifold $(\mathfrak{B}, g)$ is transitive and the center of $G$ is not discrete, then $G$ is essentially isometric.

Proof. Since the center $Z$ of $G$ is not discrete, the Lie algebra 3 of $Z$ is of positive dimension. Therefore we can take a non-zero element $Y$ of 3 . The vector field $\tilde{Y}$ on $\mathfrak{B}$, which corresponds canonically to $Y$, is left invariant by the action of $G$ because $Z$ is the center of $G$. Since $G$ is assumed to be transitive and effctive, the vector field $\tilde{Y}$ is not zero at every point of $\mathfrak{B}$. Consequently, a function $f$ on $\mathfrak{B}$ defined by

$$
f=g(\tilde{Y}, \tilde{Y})^{-1}
$$

is positive-valued. We can prove that

$$
\mathcal{L}_{\tilde{X}} f=-2 \phi_{\tilde{X}} f
$$

holds for any element $X$ of $\$$, $\$$ being the Lie algebra of $G$. Hence, by Lemma $1, G$ is essentially isometric.

We shall prove the following

THEOREM 2. Let $G$ be a transitive conformal transformation group of a Riemannian manifold. If $G$ is isomorphic onto the direct product of a vector group and a compact Lie group, then $G$ is essentially isometric.

Proof. Assume that $G$ is isomorphic onto the direct product of a vector group $R^{l}$ of dimension $l$ and a compact Lie group $K$. First suppose $l=0$. Then $G$ is compact and hence it follows from Theorem B that $G$ is essentially isometric. Next suppose $l>0$. Then, since the center $Z$ of $G$ contains the vector group $R^{l}, Z$ is not discrete. Thus, by Lemma $3, G$ is essentially isometric.

To get Theorem 3, we need the following

LEMMA 4. Let $G$ be a connected and effective isometry group of a compact and orientable Riemannian manifold $(\mathfrak{B}, g)$. Then $G$ is isomorphic onto the direct 
product of a vector group and a compact Lie group.

Proof. Consider an arbitrary function $F$ on $\mathfrak{B}$. Then we have

$$
\int_{\mathfrak{B}} \mathcal{L}_{\tilde{X}} F d v=0
$$

for any element $X$ of $\mathbb{B}$, where $d v$ is the volume element of $(\mathfrak{B}, g)$ and $\mathbb{B}$ the Lie algebra of $G$. Therefore, for any $X, Y$ and $V$ of $\mathbb{B}$, we have

$$
\int_{\mathfrak{B}} \mathcal{L}_{\tilde{X}}(g(\tilde{Y}, \tilde{V})) d v=0
$$

which reduces to

$$
\int_{\mathfrak{B}} g([\tilde{X}, \tilde{Y}], \tilde{V}) d v+\int_{\mathfrak{B}} g(\tilde{Y},[\tilde{X}, \tilde{V}]) d v=0
$$

If we put

$$
Q(X, Y)=\int_{\mathfrak{B}} g(\tilde{X}, \tilde{Y}) d v
$$

for any elements $X$ and $Y$ of $\mathbb{B}$, then $Q$ is a symmetric positive definite bilinear form on $(S \times \mathbb{B}$ because $G$ is assumed to be effective. We have, from (5),

$$
Q([X, Y], V)+Q(Y,[X, V])=0,
$$

which shows that $Q$ is an invariant form of $\$$. Hence, $\$$ is decomposed into the direct sum

$$
\mathbb{B}=3+[\mathbb{B}, \mathbb{B}],
$$

where 3 is the center of $\mathbb{B}$ and the ideal [\$S, $\mathbb{B}$ ] is semisimple and compact (see [2] for instance). Consequently, the group $G$ is isomorphic onto the direct product of a vector group and a compact Lie group.

The above proof of Lemma 4 is quite similar to the proof of the following theorem due to Barbance [1].

THEOREM C. If $(\mathfrak{B}, g)$ is a compact and orientable Riemannian manifold of dimension $>2$ and the Weyl conformal curvature tensor field is not zero at every point of $\mathfrak{B}$, then the largest connected group of conformal transformations of (B, $g$ ) is isomorphic onto the direct product of a vector group and a compact Lie group.

From Lemma 4, we have immediately the following

THEOREM 3. Let $G$ be a conformal transformation group of a compact Riemannian manifold $(\mathfrak{B}, g)$. If $G$ is essentially isometric then $G$ is isomorphic onto a vector group and a compact Lie group. 
In fact, in the case where $\mathfrak{V}$ in Theorem 3 is not orientable, by considering a double covering of $(\mathfrak{B}, g)$, which is a compact and orientable Riemannian manifold, we can get the result of Theorem 3 .

From Theorems A and 3, we have Theorem C, while Theorem 3 holds for any conformal transformation group of a compact Riemannian manifold.

The auther wishes to express here his hearty thanks to Prof. S. Ishihara who gave him valuable advices.

\section{BIBLIOGRAPHY}

[1] BARBAnce, C., Transformations conformes d'une variété riemannienne compacte. C. R. Acad. Sci. Paris 266 (1968), 149-152.

[2] Helgason, S., Differential geometry and symmetric spaces. Academic Press, New York (1962).

[3 ] Hlavatý, V., Zur Konformgeometrie. Proc. Acad. Amsterdam 38 (1935), 281286.

[4] IsHinARA, S., Groups of projective transformations and groups of conformal transformations. J. Math. Soc. Japan 9 (1957), 195-227.

FACULTY of ENGINEERING,

Kumamoto University. 